\title{
Inevitability or opportunity?
}

\author{
Gabriel S. Aldea, MD, FACS, FACC, and William K. Edmark, MD, FACS, FACC
}

\author{
From the Division of Cardiac Surgery, University of Washington, Seattle, Wash. \\ Disclosures: Authors have nothing to disclose with regard to commercial support. \\ Received for publication Nov 28, 2016; accepted for publication Dec 1, 2016; available ahead of print January 25, \\ 2017. \\ Address for reprints: Gabriel S. Aldea, MD, FACS, FACC, and William K. Edmark, MD, FACS, FACC, Cardiac \\ Surgery, University of Washington, Box 365130, 1959 NE Pacific St, Seattle, WA 98195 (E-mail: aldea@uw. \\ edu). \\ J Thorac Cardiovasc Surg 2017;153:1291-2 \\ 0022-5223/\$0.00 \\ Published by Elsevier Inc. on behalf of The American Association for Thoracic Surgery \\ http://dx.doi.org/10.1016/j.jtcvs.2016.12.002
}

Despite continued improvements in cardiac surgical outcomes, ${ }^{1}$ the invasiveness of surgical aortic valve replacement (SAVR) remains consequential, particularly in subsets of vulnerable patient populations. In this publication, Gaudiani and colleagues ${ }^{2}$ demonstrated 2 critical findings. The first was that in high-risk patients with aortic stenosis, those undergoing SAVR have a 2-fold greater rate of mortality compared with those undergoing transcatheter aortic valve replacement (TAVR; $7.9 \%$ vs $4 \%, P=.025)$ that accounts for the difference in survival that persists to 2 years. The second was that differential mortality occurred in a finite period between 31 and 120 days but was equivalent in earlier or later periods.

These outcomes are attributed to clinical and subclinical complications of the procedure itself or to the inability to reverse underlying pathology ("failure to treat and recover") that are consequential particularly in these atrisk patients. The interactions and impact of cardiovascular pathology, coexistent morbidities, and frailty on outcomes are not understood fully and are assumed to be nonlinear interdependent covariants. Accurately estimating their combined effects remains at the core of clinical decision making. As surgeons who actively participate and lead patient screening, assessments, and therapies (with both SAVR and TAVR), these sobering findings represent an opportunity to redirect a needed dialogue. Instead of casting this and other studies as an overly simplistic binary indictment or supremacy of one treatment modality over another (TAVR vs SAVR), we should embrace these therapeutic options as a complementary continuum of therapies.

Recommendations require dynamic reassessments that reflect the evolution of technologies as well as the changing specific needs of individual patients at different phases of well-being, age, activity, and disease. Future investigations should refocus on whether current outcomes and vulnerabilities are inevitable and the best we can achieve or, alternatively, whether these represent opportunities for clinically relevant improvements.

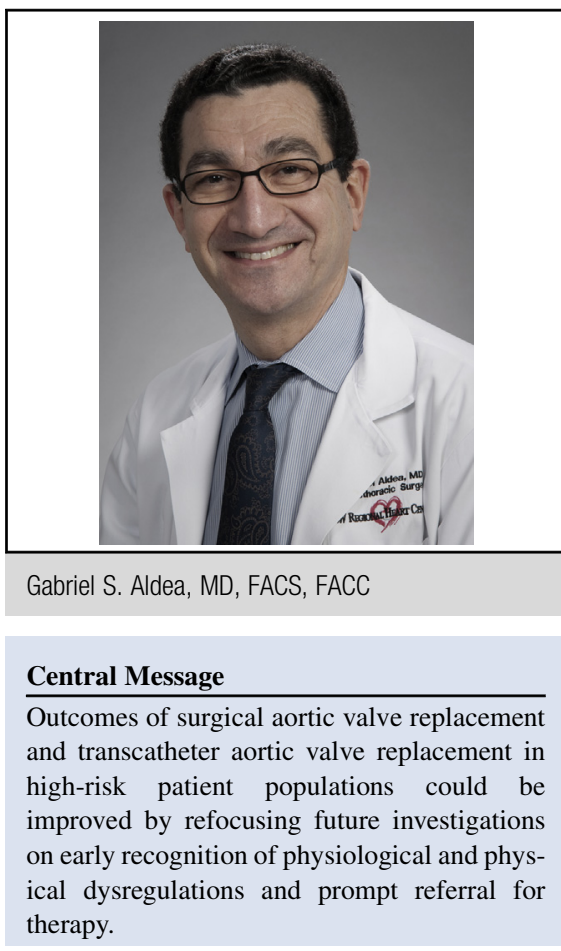

See Article page 1293.

Several areas of inquiry that aim to improve outcomes are evident:

1. Improved routine objective discernment of frailty. Highrisk patient populations are not characterized fully by the Society of Thoracic Surgeons or European System for Cardiac Operative Risk Evaluation Predicted Risk of Mortality algorithms. Physical (such as frailty and limited mobility) as well as neurologic and psychosocial challenges (such as cognitive deficits, depression, lack of autonomy, and family support) equally are impactful and relevant. When these conditions are present, TAVR should be considered strongly in an attempt to limit ANY short-term disability with its potential major adverse consequences. At its extremes of immobility, frailty, and deconditioning, these may represent hallmarks of futility. Formal routine assessment and documentation of these vulnerabilities are essential and may have broader benefit much beyond this proscribed patient population.

2. Addressing Delayed Therapy. Despite improvements in both open surgical and catheter-based treatment options, these are still perceived by patients and referring 
physicians as aggressive, invasive, and morbid. Delays in diagnosis and referral lead to physiologic decompensation and acceleration of baseline disabilities. Increased New York Heart Association class and deconditioning that progress from activity and autonomy to frailty and eventual disability significantly impact short-term risk (increased mortality by 3 -fold) as well as long-term outcomes and potential benefits. ${ }^{3,4}$ Attention to ANY change in activity level and the addition of more nuanced physiological and imaging parameters of dysregulation (such as brain natriuretic peptide and left ventricular strain) to the current reliance on symptomatic indications for referral to therapy may be beneficial and impactful. ${ }^{5,6}$ Educational initiatives are necessary to reverse the assumption that indolence and delays in referral are safe and inconsequential.

3. Reversing frailty. Frailty and debility have been assumed to be progressive, intricately linked to underlying cardiovascular pathology and inevitable. The benefit of exercise-based physical cardiac rehabilitation (CR) has been well documented in the treatment of coronary artery disease with a reduction in cardiovascular mortality and rehospitalization. ${ }^{7-10} \mathrm{CR}$ typically targets patient populations after a cardiovascular event (such as an myocardial infarction or cardiac surgery). Few studies focus on the impact of CR on valve pathology. Paradoxically, in patients with coronary artery disease, the frailest and most deconditioned (debilitated) patients, although they had the least improvement in objective measurements of physical well-being (smallest delta from baseline) compared with their more active peers, had the greatest improvement in cardiovascular mortality. Is it possible that brief, tailored physical therapy programs before a procedure (termed prehab) can improve short- and long-term outcomes regardless of the therapy used (SAVR or TAVR)?
This publication is an opportunity for our cardiovascular community to better assess and individualize valve therapies, consider the consequence of delayed therapy, and challenge the prevailing notion of the inevitability of significant morbidity and mortality from physiological and physical dysregulation in the high-risk patient.

\section{References}

1. Brennan JM, Holmes DR, Sherwood MW, Edwards FH, Carroll JD, Grover FL, et al. The association of transcatheter aortic valve replacement availability and hospital aortic valve replacement volume and mortality in the United States. Ann Thorac Surg. 2014;98: 2016-22.

2. Gaudiani V, Deeb GM, Popma JJ, Adams DH, Gleason TG, Conte JV, et al. Causes of death from the randomized CoreValue US Pivotal High-Risk Trial. J Thorac Cardiovasc Surg. 2017;153:1293-301.e1.

3. Frisoli A Jr, Ingham SJ, Paes ÂT, Tinoco E, Greco A, Zanata N, et al. Frailty predictors and outcomes among older patients with cardiovascular disease: data from Fragicor. Arch Gerontol Geriatr. 2015;61:1-7.

4. Le Tourneau T, Pellikka PA, Brown ML, Malouf JF, Mahoney DW, Schaff HV, et al. Clinical outcome of asymptomatic severe aortic stenosis with medical and surgical management: importance of STS score at diagnosis. Ann Thorac Surg. 2010;90:1876-83

5. Goodman A, Kusunose K, Popovic ZB, Parikh R, Barr T, Sabik JF, et al. Synergistic utility of brain natriuretic peptide and left ventricular strain in patients with significant aortic stenosis. J Am Heart Assoc. 2016;5(1); http://dx.doi.org/10.1161/JAHA.115.002561. pii: e002561.

6. Abramowitz Y, Chakravarty T, Jilaihawi H, Lee C, Cox J, Sharma RP, et al. Impact of preprocedural B-type natriuretic peptide levels on the outcomes after transcatheter aortic valve implantation. Am J Cardiol. 2015;116:1904-9.

7. Anderson L, Thompson DR, Oldridge N, Zwisler AD, Rees K, Martin N, et al. Exercise-based cardiac rehabilitation for coronary heart disease. Cochrane Database Syst Rev 2016;1:CD001800.

8. Lee JY, Ahn JM, Park DW, Kang SJ, Kim YH, Lee SW, et al. Impact of exercise-based cardiac rehabilitation on long-term clinical outcomes in patients with left main coronary artery stenosis. Eur J Prev Cardiol. 2016;23: 1804-13.

9. Marcassa C, Giordano A, Giannuzzi P. Five-year hospitalisations and survival in patients admitted to inpatient cardiac rehabilitation after cardiac surgery. Eur J Prev Cardiol. 2016;23:1609-17.

10. Lawler PR, Filion KB, Eisenberg MJ. Efficacy of exercise-based cardiac rehabilitation post-myocardial infarction: a systematic review and meta-analysis of randomized controlled trials. Am Heart J. 2011;162: $571-84$. 